\title{
Robust Study and Evaluate the BER Performance of Multiuser Massive MIMO Systems
}

\author{
${ }^{* 1}$ Patteti Krishna, ${ }^{2}$ B.Balaji, ${ }^{3}$ Tipparti Anil Kumar \\ ${ }^{* 1}$ Department of Electronics and Communication Engineering, Malla Reddy Engineering College, \\ Secunderabad, India, ${ }^{2}$ Department of Electronics and Communication Engineering \\ KL University, Vaddeswaram, Guntur, Andhrapradesh India, ${ }^{3}$ Department of Electronics and Communication \\ Engineering, CMR College of Engineering \& Technology, Kandlakoya, Hyderabad, Telangana, India \\ Email:*1kpatteti@gmail.com, ${ }^{3}$ tvakumar2000@yahoo.co.in
}

Received: 20 ${ }^{\text {th }}$ December 2017, Accepted: 20th January 2018, Published: 28th February 2018

\begin{abstract}
The need for higher data rates led researchers to propose another technique called a multiuser massive MIMO system that offers a larger bandwidth compared to the present wireless communication systems. In addition, significant improvement in communications quality of service (QOS), energy and spectral efficiencies and in the reduction of the cost is expected in Massive MIMO. The performance of linear detection schemes used for the uplink and downlink of a single-cell massive multi user MIMO systems in the lower frequency bands. The main objective of this paper is to study and evaluate the minimize bit error rate (BER) performance of a Multiuser massive MIMO system by using linear detection schemes, such as zero forcing precoding (ZF), Maximum-Ratio Transmission (MRT) and Minimum Mean-Square Error (MMSE)
\end{abstract}

Keywords: BER; MIMO; MU-MIMO; Linear receivers

\section{Introduction}

In upcoming years, the amount of data traffic in wireless communication will increase considerably; therefore, a new generation network, $5 \mathrm{G}$, has to be developed to increase the data capacity 1000 times compared to current 4G system [3]. Energy efficiency and faster communication response time are also expected in the future network [3]. In order to increase the spectral efficiency, you need to have one of the following options:

$>$ Very large number of base station antennas [1-2] [5-7].

$>$ Small cells.

$>$ In order to support more users, increasing the bandwidth by using the high frequency bands (millimeter Wave) is a very good choice.

In massive MIMO for example, the industry is trying to increase the number $N_{t}$ of BS antennas to 100 or more in order to simultaneously serve a large number of users $K$, say tens, with single or multiple antennas, in the same frequency band [1]. In addition, small cells are also expected in massive MIMO [6]. The channel state information $H$ is the channel propagation matrix between the $K$ users and
BS antennas array. In general, the channel propagation is modeled as a Rayleigh or Rician fading channel. In practice, the channel matrix has to be estimated by using orthogonal pilot sequences in the uplink transmission [6].

After estimating the channel state information in the uplink transmission, the BS uses the estimated channel in downlink transmission to precode the data streams to all users.

This paper can be organized as follows: SectionI briefly describes introduction of the paper, in Section-II illustrated about the system model, in Section-III and IV explains the massive MIMO uplink and downlink data transmission system, simulation results and conclusion describes in Section-V and VI.

\section{System Model}

Consider a Massive MU-MIMO BS with $N_{t}$ antennas that serves $\mathrm{K}$ single-antenna or multipleantennas users. Denote the channel coefficient from the $k^{t h}$ user to the $n^{t h}$ antenna of the BS as $h_{k, n}$ in the uplink case, which is equal to a complex small-scale fading factor times an amplitude factor that accounts for geometric attenuation and large-scale fading[1] [4] [2]:

$$
h_{k, n}=g_{k, n} \sqrt{d_{k}}
$$

(1)

Where $g_{k}$, and $d_{k}$ represent the complex small, and large-scale fading coefficients, respectively. The small-scale fading coefficients are assumed to be independent for each user, while the large-scale ones are the same for all the $N_{t}$ antennas but depend on the user's position [4]. Then, the channel matrix experienced by all the $\mathrm{K}$ users in the uplink scenario can be expressed as [4] [2]

$$
\left(\begin{array}{ccc}
h_{1,1} & \cdots & h_{K, 1} \\
. . & . . & . . \\
h_{1, N_{t}} & . \cdot & h_{K, N_{t}}
\end{array}\right)=G \sqrt{D}
$$




$$
\begin{aligned}
& G=\left(\begin{array}{ccc}
g_{1,1} & . . & g_{K, 1} \\
. . & . . & . . \\
g_{1, N_{t}} & . . & g_{K, N_{t}}
\end{array}\right) \\
& D=\left(\begin{array}{ccc}
d_{1} & . . & d_{1,1} \\
. . & . . & . . \\
d_{N} & . . & d_{K}
\end{array}\right)
\end{aligned}
$$

In massive MIMO setup, as $N_{t} \gg K$, there are two system protocols, which are frequency-division duplex (FDD) or time-division duplex (TDD) used for data transmission [1]. The TDD scheme is more efficient than FDD because the channel estimation in TDD is reciprocal, which means that the estimated channel in the uplink case is the same as the downlink. Therefore, the estimated channel can be used by BS to precode the data streams. However, in FDD case, the channel estimation is not reciprocal [1] [7].

\section{MASSIVE MIMO UPLINK DATA TRANSMISSION}

The data transmission in massive MIMO as mentioned above is done by implementing the uplink or downlink techniques. Uplink transmission is the scenario where the $K$ users transmit signals to the BS.

Let $S_{k}$ be the transmitted signal from the $k^{\text {th }}$ user. Since the $K$ users share the same time-frequency resources, the $N_{t_{\mathrm{x} 1}}$ received signal vector at the BS is modeled as follows $[\mathbf{6}][\mathbf{1 8}][\mathbf{1}]$

$$
y_{u}=\sqrt{P_{u}} H S+n_{u}
$$

where $P_{u}$ is the uplink transmission power, $S \in \square K \times 1$ is the transmitted symbols from $K$ users, $\quad n_{u} \in \square^{N_{t} \times 1}$ is the additive white noise vector with independent components, and $H \in \square^{N_{t} \times K}$ is the channel matrix.

With linear detection schemes at the BS, the transmitted symbols $\hat{S}$ can be detected by multiplying $y_{u}$ with the linear detection matrix $W \in \square^{N_{t} \times K}$ as follows [2][7]

$$
\hat{S}=W^{T} y_{u}
$$

Therefore, the received signal-to-interference-plusnoise ratio (SINR) of the $k^{\text {th }}$ stream is given by [7] where $w_{k}$ denotes the $k^{t^{h}}$ column of matrix $W$.

Then, the maximum achievable sum-rate is given by [18]

$$
\operatorname{SINR}_{k}=\frac{P_{u}\left|W_{k}^{T^{*}} h_{k}\right|^{2}}{P_{u} \sum_{h \neq k^{\prime}}^{k}\left|W_{k}^{T^{*}} h_{k}\right|^{2}+\left\|w_{k}\right\|^{2}}
$$

$$
R=\sum_{k=1}^{K} E\left\{\log _{2}\left(1+\operatorname{SINR}_{k}\right)\right\}
$$

(6)

where $E$ is the mean. The linear detection matrix $W$ can be designed by using one of the following techniques [7]

\section{A. Maximum ratio combining receiver (MRC)}

We set $W$ equal to $H^{*}$, which is the complex conjugate of $H$. At low SNR, MRC can achieve the same array gain as in the case of a single-user system, but it performs poorly in multiuser interference.

\section{B. Zero Forcing receiver $(Z F)$}

By contrast to MRC, zero-forcing ( $\mathrm{ZF}$ ) receivers take the multiuser interference into account, but neglect the effect of noise. The $\mathrm{ZF}$ receiver matrix is the pseudo-inverse of the channel matrix $\mathrm{H}$. With $\mathrm{ZF}$, we have

$$
W=H\left(H^{H} H\right)^{-1}
$$

where the small $H$ is the transpose conjugate

\section{Minimum Mean Square Error receiver (MMSE)}

The linear minimum mean-square error (MMSE) receiver aims to minimize the mean-square error between the estimate $W^{\prime} y_{u}$ and the transmitted signal $S$. Therefore, the MMSE receiver matrix is

$$
W=H\left(H^{H} H+\sigma^{2} I_{K}\right)^{-1}
$$

(8)

where $I_{K}$ is the identity matrix, and $\sigma^{2}$ is the variance of the noise. MMSE receiver matrix works as MRC at low SNR and as ZF at high SNR.

\section{MASSIVE MIMO DOWNLINK DATA TRANSMISSION}

In the downlink transmission scenario, the BS transmits data to all $K$ users. Let $X \in \square K \times 1$ be the transmitted symbols vector intended for all $K$ users. Then by using linear precoding technique, the precoding vector $X_{F}[2]$ [8] is

$$
X_{F}=\sqrt{\alpha} F X
$$


where $F \in \square^{N_{t} \times K}$ is the precoding matrix, and $\alpha$ is a normalization constant chosen to satisfy the power constraint $E\left\{\left\|X_{F}^{2}\right\|\right\}$. Thus [7],

$$
\alpha=\frac{1}{E\left\{t r\left(F F^{H}\right)\right\}}
$$

Therefore, the received signal at $K$ users is given by $y_{d}=\sqrt{P_{d}} H^{T} X_{F}+n_{d}$

where $P_{d}$ is the downlink transmission power, $n_{d} \in \square^{K \times 1}$ is a Gaussian noise vector, and $H^{T} \in \square K \times N_{t}$ is the channel matrix.

By implementing the precoding techniques above, the $S I N R_{k}$ is given as follows

$$
\operatorname{SINR}_{k}=\frac{\alpha P_{d}\left|h_{k}^{T} f_{k}\right|^{2}}{\alpha P_{d} \sum_{h \neq k^{\prime}}^{k}\left|h_{k}^{T} f_{k^{\prime}}\right|^{2}+1}
$$

where $f_{k}$ denotes the $k^{t h}$ column of matrix $F$. Thus, the maximum achievable sum-rate in the downlink scenario is given by

$$
R=\sum_{k=1}^{K} E\left\{\log _{2}\left(1+\operatorname{SINR}_{k}\right)\right\}
$$

The three linear precoders are maximum-ratio transmission (MRT) (also called conjugate beamforming), ZF, and MMSE precoders; similarly, the precoding techniques have similar operational properties as MRC, ZF, and MMSE. The equations for these precoders are as follows

$$
F=\left\{\begin{array}{cc}
H, & \text { forMRT } \\
H\left(H^{T} H\right)^{-1}, & \text { forZF } \\
H\left(H^{T} H+\frac{K}{P_{d}} I^{-1}\right)^{-1}, & \text { forMMSE }
\end{array}\right\}
$$$$
(1
$$

\section{Simulation Result}

\section{A. Single Cell Massive Multiuser MIMO System: uplink and downlink}

In this section, we provide a set of performance results for uncoded QPSK uplink and downlink transmission in the lower frequency bands with different numbers of BS antennas, $N_{t}$, serving $K=10$ users. The channel is also considered here to be a quasi-static flat Rayleigh fading and it is modeled by small-scale fading only, not considering large-scale variations. Because for a small cell, the distance is small enough so large-scale variation can be ignored. In addition, the BS has a perfect knowledge of the channel to detect and precode the data. The following performance results show the BER versus the received $\frac{E_{b}}{N_{0}}$. Figure 1 shows the BER performance results for uplink and downlink transmission with $N t=50$ and $K=10$. The performance of the MRC/MRT, ZF, and MMSE are compared to each other. As can be seen from the plot, MMSE and ZF detector/precoder perform significantly better than MRC/MRT over the higher range of $\frac{E_{b}}{N_{0}}$. Due to their sensitivity to multi user interference, MRC/MRT has the worst performance over the higher range of $\frac{E_{b}}{N_{0}}$. In the lower $\frac{E_{b}}{N_{0}}$ regime, MRC/MRT performs similarly to the other schemes. The overall BER performance of the downlink transmission is worse than the uplink one due to the normalization constant chosen to satisfy the specific power constraint. However, a bit error probability of $10^{-6}$ can be obtained with $\frac{E_{b}}{N_{0}} \approx 5$ $d B$ only using either ZF or MMSE precoder for the downlink transmission.

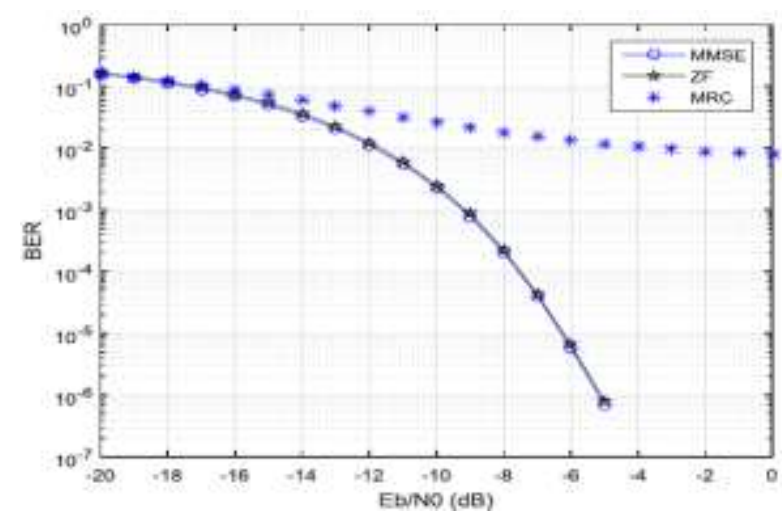

(a)uplink Transmission

When $N_{t} \gg K$, both the multi user interference and the fading effects tend to disappear, this gives clear insight about the favorable environments. Consequently, the BER performance for the massive MU-MIMO $N_{t} \times K$ is improved as can be seen in Figure 2.

It is seen from the plot that for a bit error probability of $10^{-6}$ on the uplink transmission, the $250 \times 10$ massive MU-MIMO provides about $8 d B$ gain over the use of the $50 \times 10$ uplink massive MUMIMO system. In addition, for a bit error probability 
of $10^{-6}$ on the downlink transmission, the $250 \times 10$ massive MU-MIMO provides also about $8 \mathrm{~dB}$ gain over the $50 \times 10$ downlink.

Although the MRC/MRT requires the lowest complexity among the detectors and precoders, it performs poorly; therefore, its use should be avoided in favour of the other detectors and precoders such as ZF and MMSE.

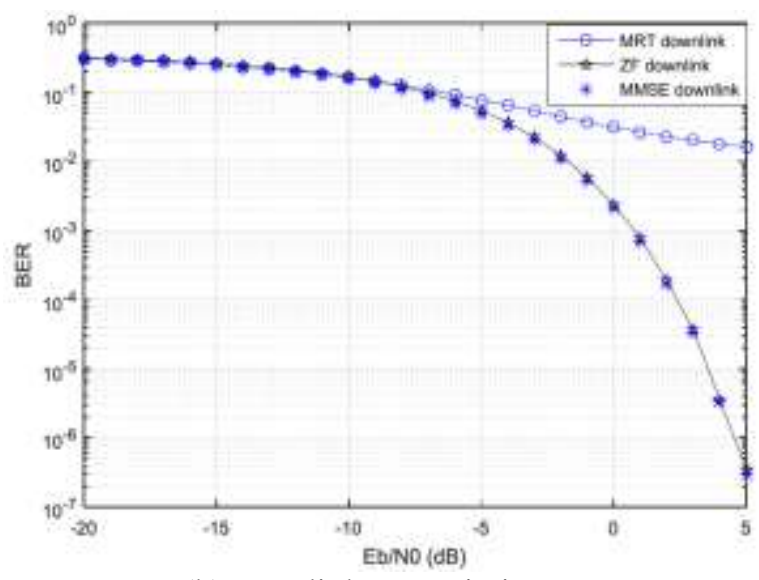

(b)Downlink transmission

Figure 1: BER performance for QPSK massive MIMO system on uplink and downlink transmission with $K=10$ and $N_{t}=50$.

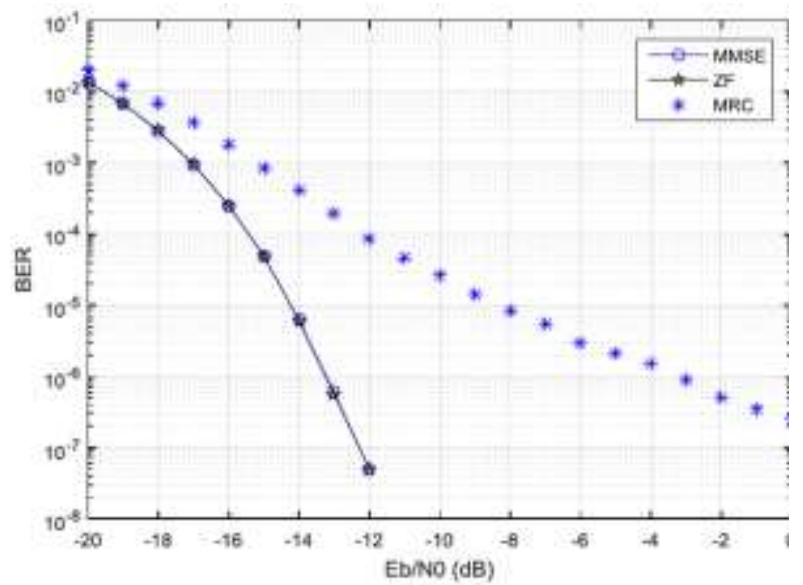

(a) Uplink Transmission

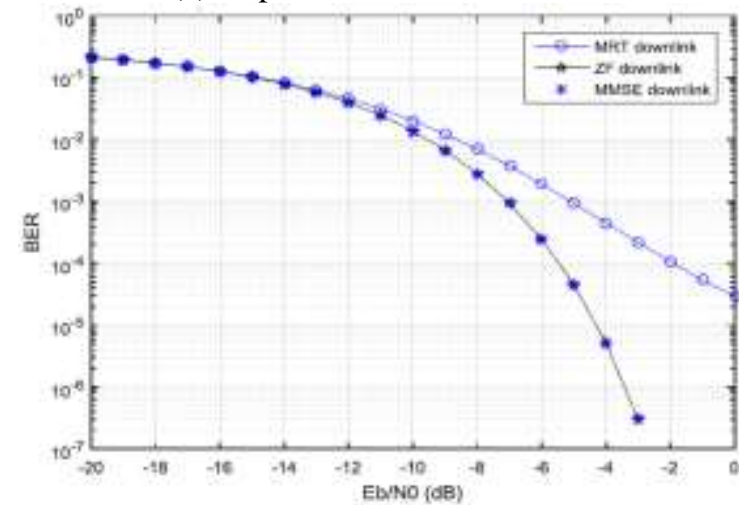

(b) Downlink Transmission

Figure 2: BER performance for QPSK massive MIMO system on uplink and downlink transmission with $K=10$ and $N_{t}$ $=250$.
However, if the value of BER obtained by MRC/MRT is acceptable, with highly increased $N_{t}$, it is better to use MRC/MRT due to its lower complexity compared to the ZF and MMSE detector/precoder.

\section{Conclusion}

We investigated massive MIMO system and evaluated the performance of uplink and downlink transmission in the lower frequency bands with different number of BS antennas, $N_{t}$ serving $K=10$ users. Although the MRC/MRT requires the lowest complexity among the detectors and precoders such as ZF and MMSE, it performs poorly. However, the BER obtained by MRC/ MRT decreased and might be acceptable by significantly increasing the number of BS antennas $N_{t}$; therefore, it is better to use MRC/MRT due to its lower complexity compared to the ZF and MMSE detector/precoder when the number of antennas is high.

\section{References}

[1] T. L. Marzetta, "Non cooperative cellular wireless with unlimited numbers of base station antennas," IEEE Commun, vol. 9, no. 11, pp. 3590-3600, Nov. 2010.

[2] LuLu, G.Y.Li, L.Swindlehurst, A.Ashikhmin, and R.Zhang, "An overview of massive mimo: benefits and challenges", IEEE Journal In Signal Process, vol. 8, No 5, October 2014.

[3] T.Endeshaw and L.Bao Le," Massive MIMO and Millimeter Wave for 5G Wireless HetNet: Potentials and Challenges", IEEE Vehicular Technology Magazine, arXiv:1510.06359v1 21 Oct 2015

[4] F.A.P.de Figueiredo, J. P. Miranda, "Uplink Performance Evaluation of Massive MUMIMO Systems", Research and Development Center on Telecommunications, Brazil, arXiv:1503.02192v1 , 7 Mar 2015

[5] E.G.Larsson, S.O.Edfors, F.Tufvesson, and B.Labs "Massive MIMO for next Generation Wireless Systems", IEEE Communication Magazine Feburary 2014

[6] K.Hosseini, J.Hoydis, S.Brink and M.Debbah “ Massive MIMO and Small Cells: How to Densify Heterogeneous Networks", IEEE ICC 2013

[7] N.Q.Hien, "Fundamentals and System Designs", Linköping Studies in Science and Technology Dissertations, No. 1642, Linköping University, 2015.

[8] T.S.Rappaport, S.Sun, R.Mayzus, H.Zhao, Y.Azar, K.Wang, G.Wong, J. K. Schulz, M. Samimi and F.Gutierrez," Millimeter Wave Mobile Communications for 5G Cellular: It Will Work!" Nyu wireless, May 29, 2013 